\title{
Über das Nucleoproteid der Schweinsleber.
}

\section{Von}

Dr. Vittorio Scaffidi, Assistent am Institute für allgemeine Pathologie zu Neapel.

(Aus der chemischen Abteilung des pathologischen Instituts der Universität zu Berlin und dem Institut für allgemeine Pathologie zu Neapel.)

(Der Redaktion zugegangen am 9. Dezember 1908.)

Hammarsten ${ }^{1}$ ) stellte aus dem Pankreas, mittels andauerndem Auskochen und Fällung des Filtrates mit Essigsäure, eine Substanz dar, welche die Eigenschaften des Nucleoproteids aufweist.

Wohlgemuth ${ }^{2}$ ) untersuchte später, mit der gleichen Methode, das Nucleoproteid der Rinderleber. Er erhielt von $1 \mathrm{~kg}$ Leber durchschnittlich 3-4 g einer weißlichen oder bräunlichen Substanz mit einem Gehalt an Phosphor von 2,5-3\%.

Schmiedeberg ${ }^{3}$ ) stellte aus der Leber von Schweinen mit einer der von Hammarsten und Wohlgemuth angewandten ähnlichen Methode eine Substanz dar, die sich durch einen hohen Eisengehalt (ca. 6\%) auszeichnete. Er legte derselben den Namen Ferratin bei.

Nach Beccari ${ }^{4}$ ) aber würde das Ferratin die Nucleoproteidgruppe darstellen, welche sich unter der Einwirkung der Hitze von Lebernucleoproteiden abspaltet und in der Brühe in Lösung bleiben würde. Er fand in demselben 1,62\% $\mathrm{Fe}$ und $2,23 \% \mathrm{P}$.

1) Hammarsten, Diese Zeitschrift, Bd. XIX, 1894.

2) Wohlgemuth, Diese Zeitschrift, Bd. XXXVII, S. 475 und Bd. XLII, S. 519.

3) Schmiedeberg, Archiv für experimentelle Pathologie und Pharmak., Bd. XXXIII, 1894, S. 101.

4) Beccari, Lo Sperimentale, 1902. 
Ich habe ${ }^{1}$ ) aus der Kaninchenleber ein Nucleoproteid dargestellt, das im Durchschnitt $0,26 \%$ Fe enthält. Der Gehalt an Eisen stieg nach Verabreichung einer organischen Eisenverbindung (paranucleinsaures Eisen $=$ Triferrin) bis zu einem Mittel von $0,66 \%$. Der Gehalt an $\mathrm{P}$ schwankte sowohl unter normalen Verhältnissen, als nach Fütterung mit Eisen innerhalb enger Grenzen, mit einem Mittel von 2,67\%.

Die von mir in vorliegenden Untersuchungen befolgte Methode, um das Nucleoproteid darzustellen, ist diejenige, welche Hammarsten für die Darstellung des Nucleoproteids aus dem Pankreas, Wohlgemuth und ich aus der Rinder- resp. Kaninchenleber angewandt haben. Ich verwendete Schweinsleber und zweimal Rinderleber.

Die Leber wurde fein zerhackt, mit dem 2-3fachen Volumen Wasser 2- bis 3 mal gekocht; die Brühe wurde abgekühlt, filtriert und mit einer geringen Menge verdünnter Essigsäure versetzt. Es fiel eine Substanz von meist flockigem Aussehen aus. Diese wurde auf einem Filter gesammelt, mit Wasser, $90 \%$ igem Alkohol, Alkohol abs. und Äther gewaschen. Einige Male wandte ich, wie weiter unten ersichtlich, zum Ausfällen sțatt verdünnter Essigsäure Weinsäurelösung an, wie sie schon von Schmiedeberg benutzt worden ist. Bei andern Versuchen habe ich den auf dem Filter gesammelten und mit Alkohol entwässerten Niederschlag mit verdünntem Ammoniak wieder in Lösung gebracht und dann nochmals mit Essigsäure resp. Weinsäure ausgefällt.

Man erhält so aus der Schweinsleber eine weißliche bis bräunliche Substanz, die nach Reinigung mittels $\mathrm{NH}_{3}$ rot-bräunlich wird. Sie ist leicht hygroskopisch und enthält ca. 12\% Stickstoff, eine Phosphorgruppe und eine Puringruppe; sie zeigt mit Orcinsalzsäure und Phloroglucinsalzsäure die Reaktion der Pentose, hat also die Eigenschaften eines Nucleoproteids. Die Substanz enthält immer Eisen in etwas schwankenden Mengen.

In den hier folgenden Tabellen sind die Ergebnisse der Analysen zusammengestellt, welche ich an den von mir, wie oben angegeben, dargestellten Nucleoproteiden vorgenommen

1) Scaffidi, Diese Zeitschrift, Bd. LIV, S. 448. 
habe, mit hauptsächlicher Berücksichtigung des in ihnen enthaltenen Eisens und Phosphors.

Der Phosphor ist in der Form von Magnesiumpyrophosphat, das Eisen in der von Ferriphospat bestimmt worden.

In der Tabelle I sind die Ergebnisse einer ersten Serie von Versuchen dargestellt, bei welcher Fe und P in dem Schweinslebernucleoproteid, welches mittels Essigsäure ausgefällt worden war, bestimmt worden sind.

Tabelle I.

\begin{tabular}{|c|c|c|c|c|c|c|}
\hline & $\begin{array}{l}\text { Ver- } \\
\text { such }\end{array}$ & $\begin{array}{l}\text { Menge des } \\
\text { analysierten } \\
\text { Nucleo- } \\
\text { proteids } \\
\text { g }\end{array}$ & $\begin{array}{l}\text { Gesamt- } \\
\text { menge } \\
\text { des } \\
\text { Eisens } \\
\text { mg }\end{array}$ & \begin{tabular}{|} 
Gesamt- \\
menge \\
des \\
Phosphors \\
mg
\end{tabular} & $\begin{array}{c}\text { Eisen } \\
\%\end{array}$ & $\begin{array}{c}\text { Phos- } \\
\text { phor } \\
\%\end{array}$ \\
\hline \multirow{5}{*}{$\begin{array}{l}\text { Schweinsleber- } \\
\text { nucleoproteid }\end{array}$} & 1 & 0,5260 & 3,45 & 12,25 & 0,65 & 2,32 \\
\hline & 2 & 0,8530 & 4,7 & 22,8 & 0,55 & 2,67 \\
\hline & 3 & 0,3310 & 6,4 & 10,4 & 1,93 & 3,18 \\
\hline & 4 & 0,4448 & 3,1 & 15,0 & 0,63 & 3,09 \\
\hline & $\tilde{5}$ & 0,3745 & 6,3 & 9,8 & 1,68 & 2,61 \\
\hline Rinderleber- & 6 & 0,3228 & 0,63 & 9,45 & 0,19 & 2,92 \\
\hline nucleoproteid & 7 & 0,6547 & 1,2 & 19,5 & 0,18 & 2,94 \\
\hline
\end{tabular}

Aus dieser Tabelle ist ersichtlich, daß mit der angegebenen Methode aus der Schweinsleber ein Körper dargestellt wird, dessen Eisengehalt von $0,55 \%$ bis zu 1,93\% schwankt und der 2,32 bis $3,18 \%$ Phosphor enthält.

In dem auf die gleiche W.eise dargestellten Nucleoproteid der Rinderleber findet man 0,18 bis $0,19 \%$ Fe und 2,92 bis $2,94 \% \mathrm{P}$, also Mengen dieses letzteren Körpers, die den von Wohlgemuth aufgefundenen entsprechen.

Während die Menge des in der Substanz enthaltenen P innerhalb ziemlich enger Grenzen schwankt, sind die Schwankungen des in derselben enthaltenen $\mathrm{Fe}$ recht bedeudend: von einem Minimum von $0,55 \% \mathrm{zu}$ einem Maximum von 1,93\%.

Einen höheren Prozentgehalt an Fe weist die Substanz auf, wenn sie mit verdünntem $\mathrm{NH}_{3}$ wieder gelöst und dann abermals mit Essigsäure ausgefällt worden ist. 
Tabelle II.

\begin{tabular}{|c|c|c|c|c|c|c|}
\hline & $\begin{array}{l}\text { Ver- } \\
\text { such }\end{array}$ & $\begin{array}{l}\text { Menge des } \\
\text { analysierten } \\
\text { Nucleo- } \\
\text { proteids } \\
\text { g }\end{array}$ & $\begin{array}{l}\text { Gesamt- } \\
\text { menge } \\
\text { des } \\
\text { Eisens } \\
\text { g }\end{array}$ & \begin{tabular}{|c|} 
Gesamt- \\
menge \\
des \\
Phosphors \\
mg
\end{tabular} & $\begin{array}{c}\text { Eisen } \\
\%\end{array}$ & $\begin{array}{c}\text { Phos- } \\
\text { phor } \\
0 / 0\end{array}$ \\
\hline \multirow{4}{*}{$\begin{array}{l}\text { Mit } \mathrm{NH}_{3} \text { ge- } \\
\text { reinigtes } \\
\text { Schweinsleber- } \\
\text { nucleoproteid }\end{array}$} & 1 & 0,1613 & 5,8 & 4,4 & 3,59 & 3,32 \\
\hline & 2 & 0,4638 & 4,7 & 13,1 & 0,93 & 2,82 \\
\hline & 3 & 0,3481 & 7,3 & 10,7 & 2,09 & 3,07 \\
\hline & 4 & 0,4439 & 6,8 & 14,3 & 1,53 & 3,22 \\
\hline
\end{tabular}

Man sieht aus Tabelle II, daß bei diesem letzteren Vorgehen der Prozentgehalt an Eisen auf 3,59 ansteigt; die großen Schwankungen im Eisengehalt, wie sie in den vorher besprochenen Versuchen vermerkt worden, bleiben aber auch hier bestehen; so ist in den vorliegenden Versuchen der niedrigste Prozentgehalt an Eisen 0,93. P schwankt zwischen 2,72 und $3,22 \%$.

Aus den Bestimmungen ergibt sich also, daß aus der Schweinsleber durch Auskochen und darauffolgendes Ausfällen mit Essigsäure eine Substanz dargestellt wird, welche einen Prozentgehalt an P aufweist, der ungefähr dem von Wohlgemuth für Rinderleber aufgefundenen entspricht und auch dem des Nucleoproteids der Kaninchenleber.

Was das Eisen anlangt, so zeigt dieses eine Schwankung des Prozentgehaltes, wie sie in meinen früheren Bestimmungen (l. c.) am Nucleoproteid der Kaninchenleber zutage getreten sind, wenn auch das Mittel des $\mathrm{Fe}$ in der Schweinsleber ein höheres ist.

Die Prozentzahlen für Eisen, welche ich in vorliegenden Versuchen gefunden habe, sind aber immer niedriger als die von Schmiedeberg ${ }^{1}$ ) angegebenen und auch als diejenigen, welche Marfori²) im Ferratin mittels Ausfällung durch Weinsäure bestimmt hat; diese letzteren schwanken zwischen 4 und $7 \%$ mit einem Mittel von $6 \%$.

1) Schmiedeberg, l. c.

2) Marfori, Archiv für experim. Pathol. u. Pharmak., Bd. XXIX. 
In einer anderen Serie von Versuchen habe ich dann den Prozentgehalt an Eisen und Phosphor in der Substanz bestimmt, sowohl wenn diese aus der Leberbrühe mit Essigsäure, als auch wenn sie mit Weinsäure ausgefällt worden.

In folgender Tabelle (s. Tabelle III) sind die Werte, welche sich bei diesen Bestimmungen ergaben, zusammengestellt.

Man verwendete bei all diesen Versuchen $500 \mathrm{~g}$ Schweinsleber. Diese wurde zerhackt, 3 mal ausgekocht und die Auszüge gemischt, abgekühlt und filtriert. Die Gesamtmenge der Auszüge wurde in zwei gleiche Teile geteilt und das Nucleoproteid aus dem einen mit Essigsäure, aus dem anderen mit Weinsäure ausgefällt. Die Werte einer jeden Bestimmung gelten also für das Nucleoproteid, das bei den Versuchen jeweils aus der Hälfte von $500 \mathrm{~g}$ ein und desselben Stückes Schweinsleber mit Weinsäure oder Essigsäure dargestellt worden ist.

Außer den Bestimmungen des $\mathrm{Fe}$ und des $\mathrm{P}$ in Nucleoproteid wurden immer solche für $\mathrm{Fe}$ auch in der abfiltrierten Brühe, sowohl nach Ausfällung mit Essigsäure, als mit Weinsäure vorgenommen.

Tabelle III.

\begin{tabular}{|c|c|c|c|c|c|c|c|}
\hline $\begin{array}{l}\text { Ver- } \\
\text { such }\end{array}$ & $\mid \begin{array}{c}\text { Nucleo- } \\
\text { proteid } \\
\text { aus der Hälfte } \\
\text { Brühe von } \\
50 \mathrm{~g} \text { Leber } \\
\text { ausgefällt mit }\end{array}$ & $\begin{array}{c}\text { Menge } \\
\text { des or- } \\
\text { haltenen } \\
\text { Nucleo- } \\
\text { proteids } \\
\text { g }\end{array}$ & $\begin{array}{l}\text { Eisen } \\
\text { im } \\
\text { Nucleo- } \\
\text { proteid } \\
\text { mg }\end{array}$ & $\begin{array}{l}\text { Eisen } \\
\text { im } \\
\text { Filtrat } \\
\text { mg }\end{array}$ & $\begin{array}{c}\text { Eisen } \\
\text { im } \\
\text { ganzen } \\
\text { mg }\end{array}$ & $\begin{array}{l}\text { Fe } \\
\text { im } \\
\text { Nucleo- } \\
\text { proteid } \\
\%\end{array}$ & $\begin{array}{c}\text { P } \\
\text { im } \\
\text { Nucleo- } \\
\text { proteid } \\
\%\end{array}$ \\
\hline \multirow{2}{*}{1} & Essigsäure & 0,735 & 10,5 & 11,4 & 21,90 & 1,43 & 2,54 \\
\hline & Weinsäure & 1,1403 & 12,53 & 8,9 & 21,43 & 1,1 & 2,45 \\
\hline \multirow{2}{*}{2} & Essigsäure & 0,8999 & 18,36 & 6,4 & 24,76 & 1,93 & 2,88 \\
\hline & Weinsäure & 1,0147 & 19,47 & 4,4 & 23,87 & 1,93 & 2,95 \\
\hline \multirow{2}{*}{3} & Essigsäure & 0,8420 & 5,38 & 9,6 & 14,98 & 0,63 & 2.94 \\
\hline & Weinsäure & 0,8404 & 4,75 & 10,7 & $1 \check{0,45}$ & 0,54 & 3,00 \\
\hline \multirow[t]{2}{*}{4} & Essigsäure & 0,4279 & 15,15 & 7,3 & 22,45 & 3,54 & 3,24 \\
\hline & Weinsäure & 0,5873 & 14,34 & $8, \dot{x}$ & 22,74 & 2,44 & $\left.3,13^{1}\right)$ \\
\hline
\end{tabular}

1) Die Werte des Versuchs 4 gelten für mit $\mathrm{NH}_{3}$ gereinigtes Nucleoproteid. 
Die Menge des Nucleoproteids, das aus je $250 \mathrm{~g}$ ein und desselben Stückes Schweinsleber mit Essigsäure oder Weinsäure ausgefällt werden konnte, ist nur einmal gleich gewesen. Sie ist in dem ersten Versuche für die mit Weinsäure ausgefällte Substanz beträchtlich größer, und auch in dem zweiten und vierten Versuche bleibt sie bei Ausfällung mit Weinsäure etwas höher.

Aus den Mittelwerten ist ersichtlich, daß man bei Ausfällung mittels Weinsäure ca. 3,5-4,5 g Nucleoproteid pro Kilo Leber erhält, während die Ausfällung mit Essigsäure nur 3 bis $3,7 \mathrm{~g}$ pro Kilo Leber liefert.

Auch der Prozentgehalt an Eisen im Nucleoproteid, das aus der gleichen Menge des gleichen Leberstückes dargestellt worden, ist verschieden, je nachdem man das Nucleoproteid mit Essigsäure oder mit Weinsäure ausgefällt hat. In diesem letztern Falle (d. h. bei Ausfällung mittels Weinsäure) findet man im allgemeinen einen kleineren Prozentgehalt an Fe, und zwar ist er geringer als der des Nucleoproteids, das mit Essigsäure ausgefällt worden ist, obwohl die absolute Menge des Eisens im Nucleoproteid nach Ausfällung mit Weinsäure in zwei Versuchen (1 und 2) höher ist, als bei der Ausfällung mit Essigsäure.

Bestimmungen des Eisens in den bezüglichen Filtraten zeigen, daß wechselnde Mengen des Metalls in Lösung bleiben.

Der Phosphor schwankt innerhalb der gewöhnlichen Grenzen, wie sie schon in den vorhergehenden Bestimmungen aufgefunden worden sind. Aus dem Vergleiche der Werte eines jeden Versuches ergibt sich, daß das Nucleoproteid der gleichen Leber sowohl, wenn die Ausfällung mittels Essigsäure, als auch wenn sie mittels Weinsäure vorgenommen worden ist, annähernd gleiche Prozentzahlen für $\mathrm{P}$ aufweist.

Wenn man aber das Nucleoproteid mit verdünntem $\mathrm{NH}_{3}$ löst und dann abermals mit Essigsäure resp. Weinsäure ausfällt, zeigt es sich reicher an Eisen und auch sein P-Gehalt steigt, wenn auch in geringerem Maße.

In folgender Tabelle IV sird die Prozentzahlen für Fe und $\mathbf{P}$ zusammengestellt, wie sie sich aus den Bestimmungen an ein und demselben Nucleoproteid ohne oder nach Reinigung mit $\mathrm{NH}_{3}$ ergeben haben. 
Es wurde bei diesen Versuchen folgendermaßen vorgegangen: von dem aus der in zwei Teile geteilten Brühe ein und desselben Leberstückes, mit Essigsäure resp. mit Weinsäure ausgefällten Nucleoproteid wurde eine gewisse Menge mit verdünntem $\mathrm{NH}_{3}$ gelöst. Die Lösung wurde filtriert, aus dem Filtrat abermals das Nucleoproteid ausgefällt und zwar jeweils, wie bei den ersten Ausfällungen, mit Essigsäure oder mit Weinsäure.

Die so gesammelte Substanz wurde mit Wasser, Alkohol von $90 \%$, Alkohol absolut. und Äther gewaschen.

Tabelle IV.

\begin{tabular}{|c|c|c|c|c|c|}
\hline $\begin{array}{l}\text { Ver- } \\
\text { such }\end{array}$ & Nucleoproteid & $\begin{array}{l}\text { Menge des } \\
\text { analysierten } \\
\text { Nucleo- } \\
\text { proteids } \\
\text { g }\end{array}$ & $\begin{array}{l}\text { Gesamt- } \\
\text { menge } \\
\text { des } \\
\text { Eisens } \\
\text { mg }\end{array}$ & $\begin{array}{l}\mathrm{Fe} \\
\%\end{array}$ & $\begin{array}{l}\text { P } \\
\% / 0\end{array}$ \\
\hline \multirow[t]{2}{*}{1} & ungereinigtes & $0,8 \check{30}$ & 4,7 & 0,55 & 2,67 \\
\hline & mit $\mathrm{NH}_{3}$ gereinigtes & $0,35 \overline{78}$ & 3,4 & 0,95 & 3,27 \\
\hline \multirow[t]{2}{*}{2} & ungereinigtes & 0,5053 & 9,7 & 1,91 & 2,95 \\
\hline & mit $\mathrm{NH}_{3}$ gereinigtes & 0,3714 & 8,5 & 2,30 & 3,5 \\
\hline \multirow[t]{2}{*}{3} & ungereinigtes & 0,4082 & 4,0 & 0,96 & 2,65 \\
\hline & mit $\mathrm{NH}_{3}$ gereinigtes & 0,3796 & 5,4 & 1,42 & 2,89 \\
\hline \multirow[t]{2}{*}{4} & ungereinigtes & 0,3990 & $\tilde{0}, 7$ & 1,43 & 2,54 \\
\hline & mit $\mathrm{NH}_{3}$ gereinigtes & 0,3885 & 7,3 & 1,88 & 3,45 \\
\hline
\end{tabular}

Aus dieser Tabelle IV ist ersichtlich, wie die Eisengehalte des Nucleoproteids sich verändern, je nachdem man die Substanz mit $\mathrm{NH}_{3}$ reinigt oder nicht.

Der Prozentgehalt an Fe nimmt nach erfolgter Reinigung immer zu. Es ist aus den in der Tabelle zusammengestellten Werten ersichtlich, daß das Mittel der Prozentzahlen für Fe im Nucleoproteid vor der Reinigung 1,21 ist, nach der Reinigung aber auf 1,60 ansteigt.

Es steigen auch die Prozentzahlen des $\mathrm{P}$ im gereinigten Nucleoproteid gegenüber denjenigen des ungereinigten; in diesem ist das Mittel 2,7\% $\mathrm{P}$, in jenem, dem gereinigten, steigt es auf $3,28 \%$. 
Woltering ${ }^{1}$ ) hat nun auch ein Nucleoproteid aus der Leber durch Ausziehen mit Chloroformwasser und kochsalzhaltigem Chloroformwasser in der Kälte und Fällung mit Essigsäure dargestellt. Ich habe diese Versuche wiederholt, außerdem auch die Extraktion mit schwach ammoniakhaltigem Wasser angewendet.

Es wurde in folgender Weise verfahren: Ca. $600 \mathrm{~g}$ einer Schweinsleber wurden zerhackt; $200 \mathrm{~g}$ des Breies wurden mit Wasser gekocht und aus dem abgekühlten und filtrierten Auszug das Nucleoproteid mit Essigsäure ausgefällt; weitere $200 \mathrm{~g}$ wurden mit ca. $600 \mathrm{ccm}$ Chloroformwasser während 24 Stunden ausgezogen, dann die Flüssigkeit abgegossen und der Rückstand nochmals 24 Stunden in wässeriger $1 \%$ iger NaCl-Lösung gelassen. Beide Auszugsflüssigkeiten wurden zusammengegossen, filtriert und aus dem Filtrat das Nucleoproteid mit Essigsäure ausgefällt. Die letzten $200 \mathrm{~g}$ des Leberbreies wurden 24 Stunden lang in $600 \mathrm{ccm}$ leicht mit $\mathrm{NH}_{3}$ versetztem Wasser ausgezogen. Aus der abfiltrierten Flüssigkeit wurde das Nucleoproteid mit Weinsäure oder Essigsäure ausgefällt. Die Ergebnisse sind in nachfolgender Tabelle zusammengestellt.

Tabelle V.

\begin{tabular}{|c|c|c|c|c|}
\hline $\begin{array}{l}\text { Ver- } \\
\text { such }\end{array}$ & Darstellungsweise des Nucleoproteids & $\begin{array}{c}\text { Menge } \\
\text { des er- } \\
\text { haltenen } \\
\text { Nucleo- } \\
\text { proteids } \\
\text { g }\end{array}$ & $\begin{array}{c}\mathrm{Fe} \\
\mathrm{im} \\
\text { Nucleo- } \\
\text { proteid } \\
\%\end{array}$ & $\begin{array}{c}\mathrm{P} \\
\mathrm{im} \\
\text { Nucleo- } \\
\text { proteid } \\
\%\end{array}$ \\
\hline \multirow[t]{3}{*}{1} & Ausfällung (Auskochen & 0,7381 & 0,93 & 2,82 \\
\hline & mit Wein- Ausziehen (Ammoniakwasser & 0,5813 & 0,28 & 0,75 \\
\hline & säure nach mit Chloroformwasser & 0,5174 & 0,72 & 1,32 \\
\hline \multirow[t]{3}{*}{2} & Ausfällung (Auskochen & 0,6313 & 1,42 & 2,86 \\
\hline & mit Essig- $\{$ Ausziehen (Ammoniakwasser & 0,5174 & 0,35 & 0,90 \\
\hline & säure nach mit |Chloroformwasser & 0,5383 & 0,64 & 1,09 \\
\hline
\end{tabular}

Aus Tabelle V ist ersichtlich, daß das Nucleoproteid, welches mit der Kochmethode dargestellt worden, einen größeren

1) Woltering, Diese Zeitschrift, Bd. XXI. 
Prozentgehalt an Fe enthält, als die mittels Ausziehens mit Chloroform- oder Ammoniakwasser erhaltenen.

Im ersten Versuche ist im Nucleoproteid, das mittels Auskochens dargestellt worden, 0,93\% Fe enthalten, in dem durch Ausziehen der Leber in Chloroformwasser erhaltenen aber $0,72 \%$, und 0,28\% finden sich in dem durch Ausziehen in Ammoniakwasser gewonnenen Nucleoproteid. Im zweiten Versuch ergaben sich 1,4\% nach Auskochen, 0,35\% nach Ausziehen mit Ammoniakwasser, 0,64\% nach Ausziehen mit Chloroformwasser.

Phosphor ist in den beiden durch Kochen dargestellten Nucleoproteiden in annähernd gleichem Verhältnisse vorhanden wie gewöhnlich; die durch Ammoniak- resp. ChloroformwasserExtraktion gewonnenen Nucleoproteide weisen einen viel geringeren P-Gehalt auf. Es wurde ferner der Gesamtstickstoffgehalt und der Purinstickstoff der durch Auskochen und Fällung mit Essigsäure resp. Weinsäure erhaltenen Nucleoproteide bestimmt.

Die Purinkörper wurden mit Silberlösung ausgefällt. Vorher wurde das Nucleoproteid, wie Burian'1) für die Bestimmung des Purinstickstoffes in den Geweben empfiehlt, während mehrerer Stunden mit 0,5\% \%ger Schwefelsäure am Rückflußkühler gekocht und weiter behandelt. Der gesamte Stickstoff wurde nach der Methode von Kjeldahl bestimmt. Die erhaltenen Werte sind folgende:

1. Mit Essigsäure ausgefälltes Nucleoproteid.

Gesamtstickstoff: Nucleoproteid $0,5209 \mathrm{~g}=45,5 \mathrm{ccm}$ $\mathrm{n} /{ }_{10}-\mathrm{H}_{2} \mathrm{SO}_{4}=0,0637 \mathrm{~g} \mathrm{~N}=12,2 \% \mathrm{~N}$.

Purinstickstoff: Nucleoproteid $0,5443 \mathrm{~g}=14,5 \mathrm{ccm}$ $\mathrm{n} / 10-\mathrm{H}_{2} \mathrm{SO}_{4}=0,0203 \mathrm{~g} \mathrm{~N}=3,73 \% \mathrm{~N}$.

2. Mit Weinsäure ausgefälltes Nucleoproteid.

Gesamtstickstoff: Núcleoproteid $0,4311 \mathrm{~g}=35,9 \mathrm{ccm}$ $\mathrm{n} / 10-\mathrm{H}_{2} \mathrm{SO}_{4}=0,0502 \mathrm{~g} \mathrm{~N}=11,64 \% \mathrm{~N}$.

Purinstickstoff: Nucleoproteid $1,1678 \mathrm{~g}=29,2 \mathrm{ccm}$ $\mathrm{n} / 10-\mathrm{H}_{2} \mathrm{SO}_{4}=0,0408 \mathrm{~g} \mathrm{~N}=3,48 \% \mathrm{~N}$.

1) Burian u. Hall, Diese Zeitschrift, Bd. XXXVIII. 
Aus vorliegenden Untersuchungen geht folgendes hervor:'

Aus Schweinsleber wird mittels Auskochen und Ausfällung mit Essigsäure oder Ausfällung mit Weinsäure (also in diesem letztern Falle mit der gleichen Methode, die Schmie deberg für die Darstellung des Ferratins anwendet) eine Substanz dargestellt, welche die Eigenschaften des Nucleoproteids besitzt; sie enthält: Purinstickstoff $3,48 \cdot 3,73 \%$, Pentose, eine Phosphorgruppe.

Dieses Nucleoproteid hat einen Prozentgehalt an Eisen, welcher beträchtliche Schwankungen aufweist; von einem Minimum von $0,54 \mathrm{zu}$ einem Maximum, nach Reinigung mit verdünntem Ammoniak, von 3,59 .

Der Prozentgehalt des Eisens im so dargestellten Nucleoproteid ist höher als der, welchen das mit Auszieben in Ammoniak-Chloroformwasser dargestellte Nucleoproteid aufweist.

Der nur einmal unter allen vorgenommenen Bestimmungen aufgefundene maximale Prozentgehalt an Eisen (3,59\%) ist bedeutend niedriger als das von Schmiedeberg angegebene Mittel des Prozentgehaltes an Eisen des Ferratins (ca. $6 \%$ ). 